\title{
Application of additive technology for the study of resonance oscillations in structures
}

\author{
Konstantin Makarov ${ }^{1}$, Evgeny Yurchenko $^{2}$ and Elena Yurchenko ${ }^{3 *}$ \\ ${ }^{1}$ DTP Science, Sochi State University, 354000, Sochi, Sovetskaya St.26a, Russia \\ ${ }^{2}$ AP Science, Sochi State University, 354000, Sochi, Sovetskaya St.26a, Russia \\ ${ }^{3}$ AP Science, Sochi State University, 354000, Sochi, Sovetskaya St.26a, Russia
}

\begin{abstract}
The methodic of studying the vibrations of small-sized physical models using additive technology has been developed. The results of the experimental determination of the resonance frequencies for harmonic oscillations and the calculation of the phase shift for two small-scale models of a three-story reinforced concrete building with a nonrigel frame has been presented. It has been shown that the production of models on a 3-D printer makes it possible to reduce the laboriousness of their manufacture, and also to expand the possibilities for accounting of operational defects in buildings and structures derived from seismic, vibrational and other types of vibrational influences based on physical modeling.
\end{abstract}

\section{Introduction}

Knowledge of resonant frequencies of oscillations of buildings and structures is necessary for the dynamic calculation of their strength and stability under seismic and vibrational influences [1]. The definition of these frequencies in full-scale conditions is quite a laborconsuming and expensive process, especially if the buildings have complex shapes in plan and height [2 - 6]. The method of physical modeling using small-sized models is more acceptable.

However, the use of models in construction is limited by the complexity of their manufacture $[7,8]$ since the materials are plexiglas or composites. In this regard, the use of additive technologies for manufacturing models of building structures is promising $[9,10]$.

In the physical simulation of the response of models to mechanical effects, they are examined using electronic sensors and computer processing. Consequently, model materials must have the electro-elastic properties, which are necessary to automate the collection and processing of simulation results.

Thus, there are conflicting requirements - on the one hand the material of the model should be from a set available for its use on a 3-D printer, and on the other hand it should have electrically conductive properties.

It was shown in [11] that ABS possesses sufficient properties (acrylonitrile butadiene styrene is a shock-resistant technical thermoplastic resin based on the acrylonitrilebutadiene-styrene copolymer) and, consequently, models of building structures can be made of it on 3-D printers and entirely buildings and structures of almost any complexity.

*Corresponding author: wsonormalno@yandex.ru 
Harmonic excitation is widely used to determine the frequencies and forms of natural oscillations of parts or structures in technology. It has the smallest number of errors in measurements. In construction, in full-scale testing of buildings [3, 5], a microseismic background of natural and technogenic origin recorded by seismometers is used.

In this article we present the results of the experimental determination of the resonance frequencies for harmonic oscillations and the calculation of the phase shift for small-scale (scale M 1: 100) models of a three-story reinforced concrete building with a bezricel frame made of Plexiglas (handmade polymethyl methacrylate) and ABS plastic printed on 3-D printer.

\section{Materials and methods}

The materials used for modeling have the effect of electrostriction, which is used in converters for measuring the variations in the dimensions of solids.

Electrostriction is the deformation of dielectric materials in an electric field, proportional to the square of the electric field strength. It is due to the polarization, that is, the displacement under the action of an external electric field, molecules carrying electric charges or changing the orientation of the dipoles. The effect of electrostriction is quadratic, so the deformation is directly proportional to the square of the field strength, and the sign of deformation (compressed or stretched by the body under the action of the field) does not depend on the direction of the electric field.

The setup for conducting the experiments consisted of the following components:

1. The subsystem of excitation of acoustic mechanical oscillations, including:

a) sound source - personal computer Lenovo B 560 or any other mp3 player;

b) power amplifier of the audio signal (in this case - Sony Sony CFS-715S Mega Bass);

c) battery power $12 \mathrm{~V} \mathrm{7,0} \mathrm{Ah;}$

d) an acoustic system with a dynamic head 4GD-35 from an electro-player "Akordsstereo".

2. Connecting (unshielded) and signal (shielded) power wires.

3. Subsystem for recording secondary mechanical oscillations:

a) signal wires with electrodes, shielded, for the left and right channels;

b) two-channel microphone amplifier with autonomous power;

c) signal wires for connecting the line output of the microphone amplifier, with the line input of the sound card of the receiving computer.

4. Receiving personal computer, working as an oscilloscope.

During the experiments and measurements, the power supply in the laboratory was turned off for reasons of minimizing electrical interference. All units were powered by direct current from built-in batteries or accumulators.

The installation was assembled from separate blocks. The acoustic system was installed horizontally, the speaker cone up, and the magnet down. Under the corners of the acoustic system vibration isolators were placed. Between the lower edge of the speaker system and the supporting surface, an air gap equal to the thickness of the vibration isolators was left. The acoustic system was equipped with a container with dry coarse sand, simulating the ground. Thus, the vector of acoustic mechanical oscillations was directed along the normal from the bottom up.

Preliminary, to determine the frequency of mechanical resonance of the system, a subsystem of excitation of acoustic mechanical vibrations was assembled.

The sound source plays back files with the recording of sinusoidal oscillations of the audio frequency with a sampling rate of 1 hertz. The estimation of which of the frequencies turns out to be resonant was made visually, by a sharp increase in the amplitude of the mechanical oscillations of the building model and the soil in the vessel. The experiment 
was carried out separately for each of the models - from ABS plastic and Plexiglass. The weight of the model of ABS plastic is $104.5 \mathrm{~g}$, the mass of the model from plexiglass is $98.3 \mathrm{~g}$.

The oscillograms were recorded for given points, to which the electrodes of the signal wires for the left and right channels of the microphone amplifier are connected. Wires with electrodes were fixed so that they did not move relative to the model under investigation during the experiment.

When the mechanical vibrations of the model are excited, it becomes the source of weak electromagnetic oscillations due to the electroelastic properties of the material. These oscillations are perceived by the electrodes, amplified by a microphone amplifier and are registrated by computer, which is used as a low-frequency two-channel oscilloscope. The method for determining the electric oscillations around electroelastic materials is borrowed from [13].

\section{Results}

Based on the analysis of the oscillograms from the test frequencies $25,39,40$ and $41 \mathrm{~Hz}$, low and high frequencies of the natural vibrations of the models are calculated. The maximum values of the phase shift were chosen from the resonance frequencies of the models. The choice was made according to [14]. The method is based on the relationship between the deformation (phase shift) measured in grads and the test frequency, measured in Hz. The phase shift is calculated by multiplying by $2 \pi$ - the time difference between the oscillogram of the left and right channel divided by the oscillation period of the lowest of the frequencies.

Since one-dimensional waves recorded by the oscilloscope are used in the tests, the wave spreading along the $\mathrm{x}$-axis (time axis) is a sinusoid. The wave has a length $\lambda$ and a frequency $\mathrm{f}$. Then, for $\mathrm{t}=0$, the wave is given by the expression:

$$
\mathrm{D}=\mathrm{D}_{\mathrm{M}} \sin (2 \pi x / \lambda)
$$

where $\mathrm{D}$ is the displacement of the wave profile at the point $\mathrm{x}, \mathrm{D}_{\mathrm{M}}$ is the wave amplitude.

The above expression describes the shape of a sinusoidal wave moving along the $\mathrm{x}$ axis with velocity $\mathrm{V}$. Since $\mathrm{V}=\lambda \mathrm{f}$, the formula can be rewritten in more convenient form:

where $\mathrm{T}=1 / \mathrm{f}$, or in the form:

$$
\mathrm{D}=\mathrm{D}_{\mathrm{M}} \sin [(2 \pi x / \lambda)-2 \pi \mathrm{t} / \mathrm{T}]
$$

$$
\mathrm{D}=\mathrm{D}_{\mathrm{M}} \sin (\mathrm{k} x-\omega \mathrm{t})
$$

where $\omega=2 \pi f=2 \pi / T$ is the circular frequency, and $k=2 \pi / \lambda$ is the wave number.

The expression $\mathrm{k} x$ - $\omega \mathrm{t}$ and the corresponding quantities are the phase of the wave.

A.P. Sinitsyn [15] suggested to take into account the finite speed of wave propagation and to consider the action of the traveling wave on the base and construction at separate intervals of time as it moves forward. It is also proposed to set the wave pattern by a movable load, in contrast to the stationary case when the resultant application is suddenly applied with eccentricity.

In the full-scale testing of buildings for seismic action, according to [1], E.E. Haciyan . Its propagation from the base of the building with finite velocity is taken into account and integration of the equations of motion into finite time intervals is effected. This made it possible to characterize at each stage of the system's existence by its growing number of equations of motion. Moreover, the system itself has an increasing number of degrees of freedom, hence different initial conditions will correspond to different displacements of floors, and between the inertial loads on them there will be a phase shift.

The values of resonant frequencies and phase shifts are shown in Figures 1,2 . 


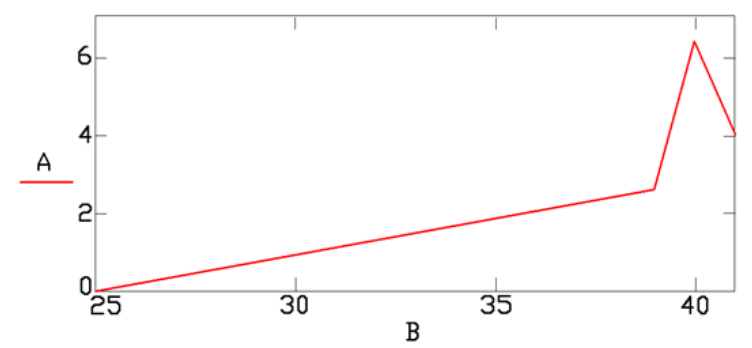

Fig. 1. Phase shift A (range) at experimental frequenses B $(\mathrm{Hz})$ for plexiglass model

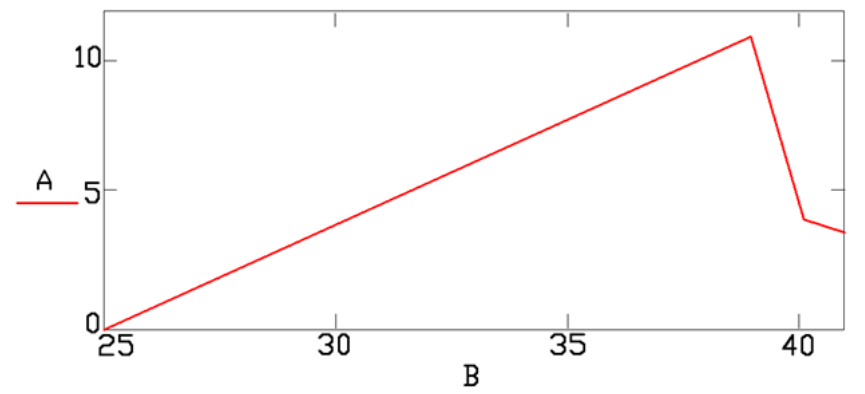

Fig. 2. Phase shift B (range) at experimental frequenses B (Hz) for model made from ABS plastic

\section{Conclusion}

1. A methodology for studying vibrations of small physical building models using additive technologies and electrostriction effect has been developed.

2. The conducted experiments allow to determine the values of:

- resonance frequencies for the model of plexiglass - $40 \mathrm{hz}$, for the model of ABS plastic $-39 \mathrm{hz}$,

- the maximum phase shift from the records of harmonic oscillations of the same frequency along the left and right channels, which characterize the resonance.

3. Manufacturing models on a 3-D printer made of ABS plastic, which has an electrostriction effect, allows to reduce the complexity of manufacturing models of buildings and structures and, accordingly, to expand the possibilities of assessing operational defects of buildings and structures from seismic, vibrational and other types of vibrational influences based on physical modeling.

\section{References}

1. E. Ye. Khachiyan., V.A . Ambartsumyan, Dynamic models of structures in the theory of seismic resistance. ( Moscow: Nauka, 203, 1981)

2. GOST 31937-2011. Interstate standard. Buildings and constructions. Rules for inspection and monitoring of technical condition.( Moscow: Standardinform, 59, 2014)

3. GOST R 54859-2011. Buildings and constructions. Determination of the parameters of the fundamental pitch of natural oscillations.(Moscow: Standardinform, 19, 2012)

4. T.A. Belash, P.L. Rybakov Buildings with suspended structures in seismic regions ( Engineering and Construction Journal, No. 5 (65) 17-26, 2016) 
5. A.V. Patrikeev, E.K. Salatov Fundamentals of the method of dynamic monitoring of deformation characteristics of buildings and structures (Vestnik MGSU, No. 1. 133138,2013 )

6. S.A. Tatarkin, R.A. Malkov, S.I. Tsarapkin, K.V. Chekster Methodology of seismoacoustic study of strength characteristics of brickwork and reinforced concrete structures of buildings and structures (Development of cities and geotechnical construction, № 14 , 2012) https://docviewer.yandex.ru/view/431480631.

7. E.N. Peresypkin, E.A. Yurchenko, E.E. Yurchenko Experimental study of the model of a section of an apartment house of a typical series 1-335 AS under dynamic influences ( RUE "Institute of BelNIIS". Collection of scientific papers. Minsk, no. 7. 103-111, 2015,)

8. N.V. Bekrenev, I.V. Zlobina Experimental study of the forms of forced oscillations of articles made of composite materials at ultrasonic loading frequencies (Fundamental'nye issledovaniya, No. 12-1 19-24, 2017) URL: http://fundamentalresearch.ru/en/article/view?id=41973.

9. N.I. Vatin, L.I. Chumadova, I.S. Goncharov, V.V. Zykova, A.N. Karpenya, A.A. Kim, E.A. Finashenkov $3 D$ printing in construction (Construction of unique buildings and structures, No. 1 (52) , 27-46, 2017)

10. N.V. Kamonichkina, I.V. Kocheshkov Investigation of the strength characteristics of a model material obtained by the FDM-printing method // Additive Technologies, 2018. No. 3. P. 39-41.

11. K.N. Makarov, E.A. Yurchenko, E.E. Yurchenko Study of the electroelasticity of a cantilever specimen made of ABS plastic printed on a 3-D printer (Globalization of modern scientific research. International Scientific Symposium SWORD, May 29-30, 2018) https://www.sworld.com.ua/index.php/en/symposia/symposia-ru/simpoziummaterial/simpoziumarhiv/symposiumru-2018.

12. M.M. Isakovich, L.I. Kleiman, B.Kh. Perchanok Elimination of vibrations of electrical machines. ( L .: Energia, 216, 1969)

13. Yu.K. Bivin Investigation of electric fields during dynamic deformation of polymers ( Journal of Technical Physics, Vol. 80. Issue. 6. 58-63, 2010)

14. Experimental studies of the properties of materials under complex thermomechanical influences / Pod. Ed. V.E.Vildemana. (Moscow: Fizmatlit, 204, 2012)

15. A.P. Sinitsyn Practical methods for calculating structures for seismic loads. (Moscow: Stroiizdat, 144, 1967) 